\title{
Horndeski model in nonlinearly realized supergravity
}

\author{
Yusuke Yamada ${ }^{a}$ and Jun'ichi Yokoyama ${ }^{a, b, c}$ \\ ${ }^{a}$ Research Center for the Early Universe (RESCEU), Graduate School of Science, \\ The University of Tokyo, Hongo 7-3-1, Bunkyo-ku, Tokyo 113-0033, Japan \\ ${ }^{b}$ Department of Physics, Graduate School of Science, \\ The University of Tokyo, Hongo 7-3-1, Bunkyo-ku, Tokyo 113-0033, Japan \\ ${ }^{c}$ Kavli Institute for the Physics and Mathematics of the Universe (Kavli IPMU), UTIAS, WPI, \\ The University of Tokyo, Kashiwa, Chiba, 277-8568, Japan \\ E-mail: yamada@resceu.s.u-tokyo.ac.jp, \\ yokoyama@resceu.s.u-tokyo.ac.jp
}

ABSTRACT: We construct the Horndeski Lagrangian within non-linearly realized supergravity. We will show that the bosonic part of the Horndeski Lagrangian can be realized. Gravitino naturally couples to Horndeski sector in a super-covariant way. Such gravitino couplings are also free from ghosts.

KEYwords: Supergravity Models, Supersymmetric Effective Theories, Cosmology of Theories beyond the SM

ARXiv EPrint: 1906.11430 


\section{Contents}

1 Introduction 1

2 Pure de Sitter supergravity 2

3 Horndeski Lagrangian in pure de Sitter supergravity 4

$\begin{array}{lll}4 & \text { Summary } & 7\end{array}$

\section{Introduction}

One of the most important goals of contemporary physics is to construct a theory to describe the entire history of the Universe consistent with observations from its creation to the current state. One may expect the birth of the classical Universe is described by string theory which is the most promissing candidate of quantum gravity. Then physics of inflation, which is an indispensable ingredient of modern cosmology to solve the horizon and the flatness problems as well as to generate curvature perturbations [1], is plausibly described by supergravity as the low energy effective theory of superstring, since observational constraints on the tensor perturbations tell us that the energy scale of inflation is at least several orders smaller than the Planck scale (at that time) [2].

In order to realize inflation in supergravity, first of all, we must realize a (quasi) de Sitter universe which inevitably breaks supersymmetry. Recently the simplest supergravity model of de Sitter space, dubbed pure de Sitter supergravity, was proposed [3, 4], in which the physical degrees of freedom are graviton and massive gravitino. In this model, spontaneous supersymmetry breaking is realized with a constrained chiral superfield $S(x, \theta)$ satisfying the nilpotent condition $S^{2}(x, \theta)=0$ [5-7], which describes the Goldstino first found by Volkov and Akulov [8]. Such a superfield is also known to be the low energy effective description of anti-D3 brane in string theory [9-13]. The nilpotent superfield has also been applied to supergravity cosmology models e.g. in [14-18].

Turning our eyes to the bottom-up approach to inflationary cosmology, the cosmic microwave background (CMB) observations strongly favor single-field inflation as we do not find any trace of isocurvature modes [19]. Then the generalized G-inflation [20] is the theory we should consider because it is the most general single-field inflation model containing practically all the known models of single-field inflation from potential driven models including the Starobinsky model [21] to kinetically driven ones [22, 23] with or without various nonminimal couplings to gravity [24, 25]. This model is based on the Horndeski theory [26] or the generalized galileon [27] which are the most general covariant scalar-tensor theory whose field equations are of second-order so that ghost instability is absent. 
It is unanimously agreed that sensible theories should not contain fundamental ghost fields [28-30], but ghost-free nature is important even from low energy effective field theory (LEFT) viewpoint. In general, LEFT contains infinite number of derivative interactions originating from integrating heavy fields out, which would be schematically given by $f\left(\frac{\partial}{M_{H}}, \phi_{L}\right)$ where $M_{H}$ denotes heavy field mass and $\phi_{L}$ being light fields. For instance, the propagator of a heavy scalar field $M_{H}^{-2}\left(1+\partial^{2} / M_{H}^{2}\right)^{-1}$ gives rise to such interaction. All the terms are suppressed by heavy field mass and $M_{H}$ becomes the cut-off scale of LEFT. On the other hand, ghost-free type higher-derivative terms does not have any pole of heavy fields. Therefore, even if they dominate the dynamics, any heavy fields would not be excited, and ghost-free higher derivative terms can be the dominant part of dynamics as long as strong coupling regime is avoided. ${ }^{1}$ In other words, among various higher-derivative terms, only ghost-free ones can be leading order terms. Also, the ghost-free type interactions may have hidden symmetry [31, 32], which becomes manifest in the affine-metric formulation.

The purpose of this work is to construct the Horndeski model coupled to pure de Sitter supergravity, namely, to embed the Horndeski Lagrangian within nonlinearly realized supergravity to make the generalized G-inflation possible in supergravity. ${ }^{2}$ Supergravity realization of ghost free higher-derivative interaction has been a difficult issue, since supersymmetry predicts not only the desired term but also additional derivative couplings, which lead to ghost instabilities. Ghost-free higher derivatives in supersymmetric models are discussed e.g. in [41-56]. As we will show, all the issues of ghost instabilities are circumvented once supersymmetry is nonlinearly realized, and the Horndeski Lagrangian is consistently embedded within supergravity. In particular, the resultant supersymmetric Horndeski model has couplings to gravitino without ghost instabilities.

\section{Pure de Sitter supergravity}

Let us start with a review the construction of the pure de Sitter supergravity model $[3,4]$. Throughout the manuscript, we use the notation of [57].

The pure de Sitter supergravity model consists of the standard supergravity multiplet and a nilpotent chiral superfield $S(x, \theta)[5-7]$ satisfying

$$
S^{2}(x, \theta)=0 .
$$

\footnotetext{
${ }^{1}$ One may think that the use of the Horndeski Lagrangian is an approximation in LEFT: in the derivative expansion in LEFT Lagrangian, the lowest order equation of motion of a real scalar $\phi$ is given by $\partial^{2} \phi=m^{2} \phi$. Using this leading equation of motion, one can reduce the number of derivatives in the LEFT Lagrangian. This procedure is nothing but elimination of heavy particle poles appearing in LEFT Lagrangian. Such an approximation is justified as long as the energy scale we consider is smaller than the mass of integrated heavy fields $M_{H}$. After reducing all the possible additional poles, we are left with the Lagrangian which has no additional poles for a scalar and a tensor, or equivalently, the Horndeski Lagrangian. Therefore, the construction of Horndeski Lagrangian would be useful even in the LEFT viewpoint.

${ }^{2}$ One might wonder if this is a marriage of losers, as LHC does not discover supersymmetry yet, and trivial but strong constraints have been obtained against the Horndeski theory from GW170817 and GRB 170817A in terms of the propagation speed of gravitational waves [33-36] using the formula derived in [20]. (See also [37] for nontrivial analysis and [38, 39] for earlier works on the possible constraints from the propagation speed.) These arguments only apply in the low energy regime well below TeV scale and we should consider the full theories to describe physics of inflation [40].
} 
The nontrivial solution to this constraint is that the lowest component of $\left.S(x, \theta)\right|_{\theta=0}=s(x)$ is no longer an independent degree of freedom, but becomes the fermion bilinear

$$
s(x)=\frac{\chi_{S}^{\alpha} \chi_{S \alpha}}{2 F^{S}}
$$

where $\chi_{S}$ is the left handed Weyl spinor and $F^{S}$ the auxiliary component in $S(x, \theta)$. Since the auxiliary field $F^{S}$ is in the denominator in (2.2), the expectation value of $F^{S}$ should be non-vanishing everywhere. In other words, this constrained superfield can be defined only when $\left\langle F^{S}\right\rangle \neq 0$, namely supersymmetry is spontaneously broken. This is not a problem as the supersymmetry is broken in de Sitter space anyway. One may think of this constrained superfield as an effective description of sGoldstino $s(x)$ decoupling [58].

Due to the constraint on $S(x, \theta)$, the most general superpotential is

$$
W=W_{0}+\mu^{2} S
$$

where $W_{0}$ and $\mu$ are real constants. Besides that, the form of the Kähler potential is restricted to

$$
K=S \bar{S}
$$

The action of the pure de Sitter supergravity is given by

$$
\mathcal{L}=\int d^{2} \Theta 2 \mathcal{E}\left[\frac{3}{8}\left(\overline{\mathcal{D}}^{2}-8 \mathcal{R}\right) e^{-\frac{K}{3}}+W\right]+\text { h.c. }
$$

where $W$ and $K$ are defined in (2.3) and (2.4). One needs to integrate out the auxiliary complex scalars $F^{S}, M$ and vector $b_{a}$, which requires straightforward but tedious calculations. The complete component action including fermions is shown in [3, 4]. Here we will focus on the system in the unitary gauge of supersymmetry:

$$
\left.\frac{1}{\sqrt{2}} \mathcal{D}^{\alpha} S\right|_{\theta=0}=\chi_{S}^{\alpha}=0
$$

which significantly simplifies the Lagrangian. Under the unitary gauge condition, the system is simply given by the standard supergravity action with $\chi_{S}=S=0$,

$$
\mathcal{L}=-e\left[\frac{1}{2} R+\varepsilon^{k l m n} \bar{\psi}_{k} \bar{\sigma}_{l} \tilde{\mathcal{D}}_{m} \psi_{n}-W_{0}\left(\psi_{a} \sigma^{a b} \psi_{b}+\text { h.c. }\right)-\left(\mu^{2}-3 W_{0}^{2}\right)\right] .
$$

Here $R$ is the Ricci scalar and $\psi_{\mu}$ is the gravitino. Here, the physical degrees of freedom are graviton $e_{\mu}^{a}$ and massive gravitino $\psi_{\mu}$ and nothing else. The mass of gravitino $m_{3 / 2}$ is given by $m_{3 / 2}=W_{0}$. The constant part $\Lambda^{4} \equiv \mu^{2}-3 W_{0}^{2}$ corresponds to vacuum energy density. Note that if $\mu=0$ the cosmological constant can never be positive, which was the standard lore in supergravity: the pure supergravity system with graviton and gravitino can only lead to negative cosmological constant, namely, anti-de Sitter spacetime. However, with a nilpotent superfield, we can realize the system having graviton, massive gravitino and positive cosmological constant, which is the pure de Sitter supergravity. 


\section{$3 \quad$ Horndeski Lagrangian in pure de Sitter supergravity}

We now show the Horndeski model $[20,26,27]$ coupled to pure de Sitter supergravity. In the case with linearly realized supersymmetry, the couplings having derivatives more than two lead to ghosts or dynamically propagating auxiliary fields, which are obstacles to realize Horndeski type interactions. So far, the known ghost free higher-derivative interactions with linearly realized supergravity are only of the kind of $\mathcal{L}_{2}=P(X, \phi)[41,45]$ and $\mathcal{L}_{5}=$ $G^{\mu \nu} \partial_{\mu} \phi \partial_{\nu} \phi$ [43] where $X=-\frac{1}{2} \partial_{\mu} \phi \partial^{\mu} \phi$ and $G_{\mu \nu}$ is the Einstein tensor. The latter is only known in the new minimal supergravity, and more general coupling $\tilde{G}_{5}(X, \phi) G^{\mu \nu} \partial_{\mu} \phi \partial_{\nu} \phi$ without ghosts has not been found yet.

The situation is drastically different in the nonlinearly realized supersymmetry as we will show. It seems rather natural to expect that Horndeski type interaction can be realized if supersymmetry is spontaneously broken: when supersymmetry is broken at some scale, its very low energy effective theory, where the heavy superparticles decouple, would become effectively non-supersymmetric system, and Horndeski type Lagrangian can be realized in such case. As pointed out in [59], the following expression would realize (almost) arbitrary couplings within supergravity:

$$
\int d^{4} \theta E \frac{16 S \bar{S}}{\mathcal{D}^{\alpha} \mathcal{D}_{\alpha} S \overline{\mathcal{D}}_{\dot{\alpha}} \overline{\mathcal{D}}^{\dot{\alpha}} \bar{S}} \mathcal{F}\left(\mathcal{C}, \mathcal{D}_{\alpha} \mathcal{C}, \mathcal{D}_{a} \mathcal{C}\right)=\mathcal{F}\left(C, \zeta_{\alpha}, \nabla_{a} C\right)+\cdots,
$$

where $\mathcal{C}$ is a general superfield, which may also have spinor or vector indices in general, and $C=\left.\mathcal{C}\right|_{\theta=0}$. We have also defined $\left.\zeta_{\alpha} \equiv \mathcal{D}_{\alpha} \mathcal{C}\right|_{\theta=0}$. The derivative $\mathcal{D}_{a}$ is vector derivative in superspace and $\nabla_{a}=e_{a}^{m} \nabla_{m}$ denotes the spacetime derivative covariant under diffeomorphism as well as supersymmetry. The ellipses part denotes the terms including the Goldstino $\chi_{S}$, which vanish in the unitary gauge (2.6).

The coupling (3.1) is crucial to realize the Horndeski model. From the above observation, it would be enough to find the superfield function $\mathcal{F}$ which has the Horndeski couplings in the lowest component. Since the original Horndeski model is described by a single real scalar field, we consider a chiral superfield $\Phi$ satisfying the following constraint [58-63]

$$
S(\Phi-\bar{\Phi})=0,
$$

where $S$ is the nilpotent superfield. All, but $\operatorname{Re} \Phi \mid$, of components in $\Phi$ become dependent fields of $\chi_{S}^{\alpha}$ and vanish in unitary gauge, namely

$$
\Phi(x, \theta)=\phi(x) \text { (in unitary gauge). }
$$

This constrained superfield can be realized as the effective theory of broken supersymmetry where all components but $\phi$ in $\Phi$ acquire mass due to soft supersymmetry breaking.

Let us show the function $\mathcal{F}_{i}$ which corresponds to $\mathcal{L}_{i}(i=2,3,4,5)$.

$$
\begin{aligned}
& \mathcal{F}_{2}=P(\mathcal{X}, \hat{\phi}) \\
& \mathcal{F}_{3}=-G_{3}(\mathcal{X}, \hat{\phi})\left(\mathcal{D}^{a} \mathcal{D}_{a} \hat{\phi}\right) \\
& \mathcal{F}_{4}=G_{4}(\mathcal{X}, \hat{\phi}) \hat{\mathcal{R}}_{s}+G_{4 \mathcal{X}}\left[\left(\mathcal{D}^{a} \mathcal{D}_{a} \hat{\phi}\right)^{2}-\left(\mathcal{D}_{a} \mathcal{D}_{b} \hat{\phi}\right)^{2}\right] \\
& \mathcal{F}_{5}=G_{5}(\mathcal{X}, \hat{\phi}) \hat{\mathcal{G}}^{a b} \mathcal{D}_{a} \mathcal{D}_{b} \hat{\phi}-\frac{1}{6} G_{5 \mathcal{X}}(\mathcal{X}, \hat{\phi})\left[\left(\mathcal{D}^{a} \mathcal{D}_{a} \hat{\phi}\right)^{3}-3\left(\mathcal{D}^{a} \mathcal{D}_{a} \hat{\phi}\right)\left(\mathcal{D}_{b} \mathcal{D}_{c} \hat{\phi}\right)^{2}+2\left(\mathcal{D}_{a} \mathcal{D}_{b} \hat{\phi}\right)^{3}\right]
\end{aligned}
$$


where $\hat{\phi}=\frac{1}{2}(\Phi+\bar{\Phi}), \mathcal{X}=-\frac{1}{2} \mathcal{D}_{a} \hat{\phi} \mathcal{D}^{a} \hat{\phi}$, and we have defined

$$
\begin{aligned}
& \left(\mathcal{D}_{a} \mathcal{D}_{b} \hat{\phi}\right)^{2}=\mathcal{D}_{a} \mathcal{D}_{b} \hat{\phi} \mathcal{D}^{a} \mathcal{D}^{b} \hat{\phi} \\
& \left(\mathcal{D}_{a} \mathcal{D}_{b} \hat{\phi}\right)^{3}=\mathcal{D}_{a} \mathcal{D}_{b} \hat{\phi} \mathcal{D}^{b} \mathcal{D}^{c} \hat{\phi} \mathcal{D}_{c} \mathcal{D}^{a} \hat{\phi}
\end{aligned}
$$

The superfields $\hat{\mathcal{R}}_{s}$ and $\mathcal{G}^{a b}$ are given by (see [59])

$$
\begin{aligned}
\hat{\mathcal{R}}_{s}= & -3\left(\overline{\mathcal{D}}_{\dot{\alpha}} \overline{\mathcal{D}}^{\dot{\alpha}}-8 \mathcal{R}\right) \overline{\mathcal{R}}+\frac{2}{3} \mathcal{R} \overline{\mathcal{R}}+6 G_{a} G^{a}-2 \mathrm{i} \mathcal{D}_{a} G^{a} \\
\hat{\mathcal{G}}^{a b}= & -\bar{\sigma}_{b}^{\dot{\alpha} \alpha} \mathcal{D}_{\alpha} \overline{\mathcal{D}}_{\dot{\alpha}} G_{a}+\left(\frac{1}{6} \hat{\mathcal{R}}_{s}+4 \mathcal{R} \overline{\mathcal{R}}+G_{c} G^{c}\right) \eta_{a b} \\
& +2 \mathrm{i} \mathcal{D}_{b} G_{a}+2 G_{a} G_{b}+\varepsilon^{c d}{ }_{a b} \mathcal{D}_{c} G_{d}
\end{aligned}
$$

The lowest components of the superfields are as follows (in unitary gauge).

$$
\begin{aligned}
\hat{\phi} \mid & =\phi, \\
\mathcal{D}_{a} \hat{\phi} \mid & =e_{a}^{m} \partial_{m} \phi, \\
\mathcal{D}_{a} \mathcal{D}_{b} \hat{\phi} \mid & =e_{a}^{m} \nabla_{m}\left(e_{b}^{n} \partial_{n} \phi\right), \\
\mathcal{R} \mid & =-\frac{1}{6} M, \\
G_{a} \mid & =-\frac{1}{3} b_{a}, \\
\hat{R}_{s} \mid & =\hat{R}, \\
\hat{\mathcal{G}} \mid & =e_{m}^{a} e_{n}^{b} \hat{G}^{m n} .
\end{aligned}
$$

Here we emphasize that $\hat{R}$ and $\hat{G}^{m n}$ are Ricci scalar and Einstein tensor consisting of super-covariantized spin-connection $\omega_{m}^{a b}$

$$
\begin{aligned}
\omega_{m}^{a b}= & -e^{k a} \partial_{[m} e_{k]}^{b}-e^{l b} \partial_{[l} e_{m]}^{a}+e^{k a} e^{l b} e_{m c} \partial_{[k} e_{l]}^{c} \\
& -\frac{\mathrm{i}}{2} \psi_{m} \sigma^{[a} \bar{\psi}^{b]}-\frac{\mathrm{i}}{2} \psi^{[a} \sigma^{b]} \bar{\psi}_{m}-\frac{\mathrm{i}}{2} \psi^{[a} \sigma_{m} \bar{\psi}^{b]},
\end{aligned}
$$

where $A_{[a} B_{b]}=\frac{1}{2}\left(A_{a} B_{b}-A_{b} B_{a}\right)$. The second order derivative term $\mathcal{D}_{a} \mathcal{D}_{b} \hat{\phi} \mid$ also contains the spin-connection. Thus, we find that these functions $\mathcal{F}_{i}$ give the Horndeski Lagrangian for $\phi$ nontrivially coupled to gravitino $\psi_{m}^{\alpha}$. Let us show the Horndeski Lagrangian in supergravity:

$$
\begin{aligned}
\mathcal{L}= & \int d^{4} \theta E \frac{16 S \bar{S}}{\mathcal{D}^{\alpha} \mathcal{D}_{\alpha} S \overline{\mathcal{D}}_{\dot{\alpha}} \overline{\mathcal{D}}^{\dot{\alpha}} \bar{S}} \sum_{i=2}^{5} \mathcal{F}_{i} \\
= & P(X, \phi)-G_{3}(X, \phi) \nabla^{2} \phi+G_{4}(X, \phi) \hat{R}+G_{4 X}\left[\left(\nabla^{2} \phi\right)^{2}-\left(\nabla_{m} \nabla_{n} \phi\right)^{2}\right] \\
& +G_{5}(X, \phi) \hat{G}^{m n} \nabla_{m} \nabla_{n} \phi-\frac{1}{6} G_{5 X}\left[\left(\nabla^{2} \phi\right)^{3}-3\left(\nabla^{2} \phi\right)\left(\nabla_{m} \nabla_{n} \phi\right)^{2}+2\left(\nabla_{m} \nabla_{n} \phi\right)^{3}\right]
\end{aligned}
$$


where we have defined $X=-\frac{1}{2} \nabla_{m} \phi \nabla^{m} \phi,\left(\nabla_{m} \nabla_{n} \phi\right)^{2}=\nabla_{m} \nabla_{n} \phi \nabla^{m} \nabla^{n} \phi$ and $\left(\nabla_{m} \nabla_{n} \phi\right)^{3}=$ $\nabla_{m} \nabla_{n} \phi \nabla^{n} \nabla^{k} \phi \nabla_{k} \nabla^{m} \phi$. The purely bosonic part of this action is precisely the same as Horndeski model.

Although the original Horndeski action is known to be free from ghosts, the absence of the ghosts in our supersymmetric system is still nontrivial. Since the gravitino couples to Horndeski Lagrangian through $\hat{R}$ and $\hat{G}$ as well as second-order derivative terms of $\phi$, such as $\nabla^{2} \phi$, one may wonder if the higher-derivative terms for gravitinos are produced. However, we can prove the absence of ghosts as follows. For the equations of motion of $\phi$ and graviton, it is proven by a simple observation that the difference appears only in the explicit form of the spin-connection. Therefore, the equations of motion of our model are derived from that of the original Horndeski system by simply replacing the non-supersymmetric spin-connection by (3.18). One finds that there is no second order derivative term of spin-connection, which implies that the equation of motion of the gravitino is the first order. Also, our action does not lead to more-than second-order derivatives for bosons. The gravitino variation in our action is always associated with the spin-connection. As the third-order derivative terms are canceled in the equations of motion of $\phi$ and graviton $e_{m}^{a}$, one can check that the coefficients of the variation $\delta \omega_{m}{ }^{a b}$ is up to the second order in derivatives. Since there is no derivative terms of the gravitino $\psi_{m}^{\alpha}$ in the definition of the spin-connection (3.18), $\frac{\delta \omega_{m}^{a b}}{\delta \psi_{n}^{\alpha}}$ would not increase the number of derivatives. Equivalently, the gravitino equation of motion is up to the first order differential equation of fermion and up to the second order differential equation of bosons. Thus, we find that our supersymmetric Horndeski action (3.19) is free from ghosts.

Besides the pure de Sitter supergravity action (2.5), one may add the Kähler and superpotential terms of $\Phi$, but then one should take into account the fact that $\Phi$ is a constrained superfield.

We note that the nonlinear realization is supposed to be the LEFT of spontaneously broken supersymmetry, and the theory breaks down at sufficiently high-energy scale. In our case, the cut-off scale is given by the unitarity bound $E_{\text {cut }}=\sqrt{6 \sqrt{2 \pi} m_{3 / 2} M_{p l}}$, at which four-fermi coupling becomes strong [64]. Also, if gravitino mass is much below the cut-off scale, huge gravitino production may take place $[65,66]$. If we consider inflationary models based on our supersymmetric Horndeski Lagrangian, the Hubble scale during inflation should be below the cut-off scale. Such constraint gives the inequality $m_{3 / 2}>2.8 \times 10^{3} \mathrm{TeV}\left(\frac{H}{10^{13} \mathrm{GeV}}\right)$. Therefore, light superparticles would not be expected in our construction, which is very consistent with the collider experimental result so far.

We finally note that the construction of supergravity nonlinear realization is technically not unique (but physically unique). One can also construct the Horndeski Lagrangian with the formulation e.g. in [57,67] where the Goldstino superfield is given by a constrained spinor superfield. Such a formulation may realize Horndeski action more straightforwardly. In this work, we have shown the construction in terms of the constrained superfields, which are more frequently used in phenomenological and cosmological model buildings. In either formulations, the final Lagrangian should be the same physically, and in the unitary gauge, the Lagrangian (3.19) should be reproduced. 


\section{Summary}

In this work, we have shown the embedding of Horndeski Lagrangian within (nonlinearly realized) $4 \mathrm{D} \mathcal{N}=1$ supergravity. Supersymmetric higher-derivative couplings often have the issues of ghosts or dynamical auxiliary fields, which have been an obstacle for the construction of general inflationary models with derivative interactions. As we have shown, such issues could be circumvented once supersymmetry is spontaneously broken, and indeed, we have constructed the Horndeski Lagrangian with constrained superfields, which describe the low energy effective theory of broken supersymmetry. In pure de Sitter supergravity model, the physical degrees of freedom are graviton, massive gravitino, and we have added a single real scalar field $\phi$ in the Horndeski Lagrangian. The supergravity realization of Horndeski Lagrangian is achieved in (3.19) with a constrained superfield $\Phi$. It is remarkable that gravitino couples to the Horndeski scalar $\phi$ in super-covariant way. As we discussed, additional gravitino couplings do not lead to higher-derivative terms, and do not spoil the ghost-free property of the original Horndeski Lagrangian, although we naively expect fermionic ghosts at first glance. This might be understood as the consequence of supersymmetry of the gravity multiplet: since graviton is coupled to a real scalar without ghosts, its fermionic partner, gravitino, is also free from ghosts.

We are now able to construct supergravity inflation model with Horndeski Lagrangian, where the inflaton $\phi$ is super-gravitationally coupled to gravitino. In such a case, one needs to consider e.g. the gravitino production through (non-)perturbative processes, which would be calculable on the bases of the Lagrangian we have shown.

It would also be possible to realize larger class of the ghost free scalar-tensor system [68] within our framework. However, as in our case, additional gravitino couplings would show up and one needs to check whether or not such couplings lead to fermionic ghost instabilities.

Another interesting possibility is to identify the Horndeski scalar field $\phi$ on the supersymmetric 3 -brane. It has been pointed out that supersymmetric (non $\kappa$-symmetric) 3-brane describes the Volkov-Akulov Goldstino, and the matter multiplets can couple to the 3-brane $[69,70]$. Since our Horndeski interactions are given by the couplings between nilpotent superfield and scalar multiplet $\Phi$, our system might be realized in 3-brane model, which would be useful to understand the possible realization of our model within string theory.

\section{Acknowledgments}

YY would like to thank Hiroaki Tahara for useful discussion and Shuntaro Aoki for useful discussion and collaboration on related works. The work of YY is supported by Grant-inAid for JSPS Fellows (19J00494). JY was supported by JSPS KAKENHI, Grant-in-Aid for Scientific Research $15 \mathrm{H} 02082$ and Grant-in-Aid for Scientific Research on Innovative Areas $15 \mathrm{H} 05888$. 
Open Access. This article is distributed under the terms of the Creative Commons Attribution License (CC-BY 4.0), which permits any use, distribution and reproduction in any medium, provided the original author(s) and source are credited.

\section{References}

[1] K. Sato and J. Yokoyama, Inflationary cosmology: first 30+ years, Int. J. Mod. Phys. D 24 (2015) 1530025 [INSPIRE].

[2] BICEP2, KeCK ARraY collaboration, BICEP2/Keck Array x: constraints on primordial gravitational waves using Planck, WMAP and new BICEP2/Keck observations through the 2015 season, Phys. Rev. Lett. 121 (2018) 221301 [arXiv:1810.05216] [INSPIRE].

[3] E.A. Bergshoeff, D.Z. Freedman, R. Kallosh and A. Van Proeyen, Pure de Sitter supergravity, Phys. Rev. D 92 (2015) 085040 [Erratum ibid. D 93 (2016) 069901] [arXiv: 1507.08264] [INSPIRE].

[4] F. Hasegawa and Y. Yamada, Component action of nilpotent multiplet coupled to matter in 4 dimensional $\mathcal{N}=1$ supergravity, JHEP 10 (2015) 106 [arXiv:1507.08619] [INSPIRE].

[5] M. Roček, Linearizing the Volkov-Akulov model, Phys. Rev. Lett. 41 (1978) 451 [INSPIRE].

[6] E.A. Ivanov and A.A. Kapustnikov, General relationship between linear and nonlinear realizations of supersymmetry, J. Phys. A 11 (1978) 2375 [INSPIRE].

[7] U. Lindström and M. Roček, Constrained local superfields, Phys. Rev. D 19 (1979) 2300 [INSPIRE].

[8] D.V. Volkov and V.P. Akulov, Is the neutrino a Goldstone particle?, Phys. Lett. B 46 (1973) 109.

[9] R. Kallosh and T. Wrase, Emergence of spontaneously broken supersymmetry on an anti-d3-brane in KKLT dS vacua, JHEP 12 (2014) 117 [arXiv:1411.1121] [INSPIRE].

[10] E.A. Bergshoeff et al., $\overline{D 3}$ and dS, JHEP 05 (2015) 058 [arXiv:1502.07627] [INSPIRE].

[11] R. Kallosh, F. Quevedo and A.M. Uranga, String theory realizations of the nilpotent goldstino, JHEP 12 (2015) 039 [arXiv:1507.07556] [INSPIRE].

[12] I. García-Etxebarria, F. Quevedo and R. Valandro, Global string embeddings for the nilpotent goldstino, JHEP 02 (2016) 148 [arXiv:1512.06926] [INSPIRE].

[13] N. Cribiori, C. Roupec, T. Wrase and Y. Yamada, Supersymmetric anti-D3-brane action in the Kachru-Kallosh-Linde-Trivedi setup, Phys. Rev. D 100 (2019) 066001 [arXiv: 1906. 07727] [INSPIRE].

[14] I. Antoniadis, E. Dudas, S. Ferrara and A. Sagnotti, The Volkov-Akulov-Starobinsky supergravity, Phys. Lett. B 733 (2014) 32 [arXiv: 1403.3269] [InSPIRE].

[15] S. Ferrara, R. Kallosh and A. Linde, Cosmology with nilpotent superfields, JHEP 10 (2014) 143 [arXiv: 1408.4096] [INSPIRE].

[16] G. Dall'Agata and F. Zwirner, On sgoldstino-less supergravity models of inflation, JHEP 12 (2014) 172 [arXiv:1411.2605] [INSPIRE].

[17] E. McDonough and M. Scalisi, Inflation from nilpotent Kähler corrections, JCAP 11 (2016) 028 [arXiv: 1609.00364] [INSPIRE]. 
[18] R. Kallosh, A. Linde, D. Roest and Y. Yamada, $\overline{D 3}$ induced geometric inflation, JHEP 07 (2017) 057 [arXiv : 1705. 09247] [INSPIRE].

[19] Planck collaboration, Planck 2018 results. X. Constraints on inflation, arXiv:1807.06211 [INSPIRE].

[20] T. Kobayashi, M. Yamaguchi and J. Yokoyama, Generalized G-inflation: inflation with the most general second-order field equations, Prog. Theor. Phys. 126 (2011) 511 [arXiv:1105.5723] [INSPIRE].

[21] A.A. Starobinsky, A new type of isotropic cosmological models without singularity, Phys. Lett. 91B (1980) 99 [Adv. Ser. Astrophys. Cosmol. 3 (1987) 130] [InSPIRE].

[22] C. Armendariz-Picon, T. Damour and V.F. Mukhanov, k-inflation, Phys. Lett. B 458 (1999) 209 [hep-th/9904075] [INSPIRE].

[23] T. Kobayashi, M. Yamaguchi and J. Yokoyama, G-inflation: inflation driven by the Galileon field, Phys. Rev. Lett. 105 (2010) 231302 [arXiv: 1008.0603] [InSPIRE].

[24] B.L. Spokoiny, Inflation and generation of perturbations in broken symmetric theory of gravity, Phys. Lett. B 147 (1984) 39.

[25] C. Germani and A. Kehagias, New model of inflation with non-minimal derivative coupling of standard model Higgs boson to gravity, Phys. Rev. Lett. 105 (2010) 011302 [arXiv: 1003.2635] [INSPIRE].

[26] G.W. Horndeski, Second-order scalar-tensor field equations in a four-dimensional space, Int. J. Theor. Phys. 10 (1974) 363 [inSPIRE].

[27] C. Deffayet, X. Gao, D.A. Steer and G. Zahariade, From k-essence to generalised Galileons, Phys. Rev. D 84 (2011) 064039 [arXiv:1103.3260] [INSPIRE].

[28] S.M. Carroll, M. Hoffman and M. Trodden, Can the dark energy equation-of-state parameter $w$ be less than -1?, Phys. Rev. D 68 (2003) 023509 [astro-ph/0301273] [InSPIRE].

[29] R.P. Woodard, Avoiding dark energy with 1/r modifications of gravity, Lect. Notes Phys. 720 (2007) 403 [astro-ph/0601672] [INSPIRE].

[30] F. Sbisà, Classical and quantum ghosts, Eur. J. Phys. 36 (2015) 015009 [arXiv:1406.4550] [INSPIRE].

[31] K. Aoki and K. Shimada, Galileon and generalized Galileon with projective invariance in a metric-affine formalism, Phys. Rev. D 98 (2018) 044038 [arXiv:1806.02589] [INSPIRE].

[32] K. Aoki and K. Shimada, Scalar-metric-affine theories: can we get ghost-free theories from symmetry?, Phys. Rev. D 100 (2019) 044037 [arXiv: 1904.10175] [INSPIRE].

[33] T. Baker et al., Strong constraints on cosmological gravity from GW170817 and GRB 170817A, Phys. Rev. Lett. 119 (2017) 251301 [arXiv: 1710.06394] [INSPIRE].

[34] P. Creminelli and F. Vernizzi, Dark energy after GW170817 and GRB170817A, Phys. Rev. Lett. 119 (2017) 251302 [arXiv: 1710.05877] [INSPIRE].

[35] J. Sakstein and B. Jain, Implications of the neutron star merger GW170817 for cosmological scalar-tensor theories, Phys. Rev. Lett. 119 (2017) 251303 [arXiv:1710.05893] [InSPIRE].

[36] J.M. Ezquiaga and M. Zumalacárregui, Dark energy after GW170817: dead ends and the road ahead, Phys. Rev. Lett. 119 (2017) 251304 [arXiv:1710.05901] [INSPIRE]. 
[37] E.J. Copeland et al., Dark energy after GW170817 revisited, Phys. Rev. Lett. 122 (2019) 061301 [arXiv: 1810.08239] [INSPIRE].

[38] L. Lombriser and A. Taylor, Breaking a dark degeneracy with gravitational waves, JCAP 03 (2016) 031 [arXiv: 1509.08458] [INSPIRE].

[39] L. Lombriser and N.A. Lima, Challenges to self-acceleration in modified gravity from gravitational waves and large-scale structure, Phys. Lett. B 765 (2017) 382 [arXiv: 1602.07670] [INSPIRE].

[40] T. Kobayashi, Horndeski theory and beyond: a review, Rept. Prog. Phys. 82 (2019) 086901 [arXiv: 1901.07183] [INSPIRE].

[41] J. Khoury, J.-L. Lehners and B. Ovrut, Supersymmetric $P(X, \phi)$ and the ghost condensate, Phys. Rev. D 83 (2011) 125031 [arXiv: 1012.3748] [INSPIRE].

[42] J. Khoury, J.-L. Lehners and B.A. Ovrut, Supersymmetric galileons, Phys. Rev. D 84 (2011) 043521 [arXiv: 1103.0003] [INSPIRE].

[43] F. Farakos, C. Germani, A. Kehagias and E.N. Saridakis, a new class of four-dimensional $N=1$ supergravity with non-minimal derivative couplings, JHEP 05 (2012) 050 [arXiv: 1202.3780] [INSPIRE].

[44] S. Sasaki, M. Yamaguchi and D. Yokoyama, Supersymmetric DBI inflation, Phys. Lett. B 718 (2012) 1 [arXiv:1205.1353] [INSPIRE].

[45] M. Koehn, J.-L. Lehners and B.A. Ovrut, Higher-derivative chiral superfield actions coupled to $N=1$ supergravity, Phys. Rev. D 86 (2012) 085019 [arXiv:1207.3798] [INSPIRE].

[46] M. Koehn, J.-L. Lehners and B.A. Ovrut, DBI inflation in $N=1$ supergravity, Phys. Rev. D 86 (2012) 123510 [arXiv: 1208.0752] [INSPIRE].

[47] F. Farakos and A. Kehagias, Emerging potentials in higher-derivative gauged chiral models coupled to $N=1$ supergravity, JHEP 11 (2012) 077 [arXiv:1207.4767] [INSPIRE].

[48] F. Farakos, C. Germani and A. Kehagias, On ghost-free supersymmetric galileons, JHEP 11 (2013) 045 [arXiv: 1306.2961] [INSPIRE].

[49] R. Gwyn and J.-L. Lehners, Non-canonical inflation in supergravity, JHEP 05 (2014) 050 [arXiv: 1402.5120] [INSPIRE].

[50] S. Aoki and Y. Yamada, Inflation in supergravity without Kähler potential, Phys. Rev. D 90 (2014) 127701 [arXiv: 1409.4183] [INSPIRE].

[51] S. Aoki and Y. Yamada, Impacts of supersymmetric higher derivative terms on inflation models in supergravity, JCAP 07 (2015) 020 [arXiv: 1504.07023] [INSPIRE].

[52] T. Fujimori, M. Nitta and Y. Yamada, Ghostbusters in higher derivative supersymmetric theories: who is afraid of propagating auxiliary fields?, JHEP 09 (2016) 106 [arXiv: 1608.01843] [INSPIRE].

[53] T. Fujimori, M. Nitta, K. Ohashi, Y. Yamada and R. Yokokura, Ghost-free vector superfield actions in supersymmetric higher-derivative theories, JHEP 09 (2017) 143 [arXiv: 1708.05129] [INSPIRE].

[54] T. Fujimori, M. Nitta, K. Ohashi and Y. Yamada, Ghostbusters in $f(R)$ supergravity, JHEP 05 (2018) 102 [arXiv: 1712.05017] [INSPIRE].

[55] M. Nitta and R. Yokokura, Higher derivative three-form gauge theories and their supersymmetric extension, JHEP 10 (2018) 146 [arXiv: 1809.03957] [INSPIRE]. 
[56] M. Nitta and R. Yokokura, Topological couplings in higher derivative extensions of supersymmetric three-form gauge theories, JHEP 05 (2019) 102 [arXiv:1810.12678] [INSPIRE].

[57] J. Wess and J. Bagger, Supersymmetry and supergravity, Princeton University Press, Princeton U.S.A. (1992).

[58] Z. Komargodski and N. Seiberg, From linear SUSY to constrained superfields, JHEP 09 (2009) 066 [arXiv:0907.2441] [INSPIRE].

[59] F. Farakos, S. Ferrara, A. Kehagias and D. Lüst, Non-linear realizations and higher curvature supergravity, Fortsch. Phys. 65 (2017) 1700073 [arXiv: 1707.06991] [INSPIRE].

[60] S. Ferrara, R. Kallosh and J. Thaler, Cosmology with orthogonal nilpotent superfields, Phys. Rev. D 93 (2016) 043516 [arXiv: 1512.00545] [InSPIRE].

[61] J.J.M. Carrasco, R. Kallosh and A. Linde, Minimal supergravity inflation, Phys. Rev. D 93 (2016) 061301 [arXiv:1512.00546] [INSPIRE].

[62] G. Dall'Agata and F. Farakos, Constrained superfields in supergravity, JHEP 02 (2016) 101 [arXiv: 1512.02158] [INSPIRE].

[63] S. Ferrara, R. Kallosh, A. Van Proeyen and T. Wrase, Linear versus non-linear supersymmetry, in general, JHEP 04 (2016) 065 [arXiv: 1603.02653] [INSPIRE].

[64] R. Casalbuoni et al., When does supergravity become strong?, Phys. Lett. B 216 (1989) 325 [Erratum ibid. B 229 (1989) 439] [INSPIRE].

[65] F. Hasegawa et al., Gravitino problem in minimal supergravity inflation, Phys. Lett. B 767 (2017) 392 [arXiv: 1701.03106] [INSPIRE].

[66] F. Hasegawa, K. Nakayama, T. Terada and Y. Yamada, Gravitino problem in inflation driven by inflaton-polonyi Kähler coupling, Phys. Lett. B 777 (2018) 270 [arXiv: 1709.01246] [INSPIRE].

[67] S. Samuel and J. Wess, A superfield formulation of the nonlinear realization of supersymmetry and its coupling to supergravity, Nucl. Phys. B 221 (1983) 153 [INSPIRE].

[68] D. Langlois and K. Noui, Degenerate higher derivative theories beyond Horndeski: evading the Ostrogradski instability, JCAP 02 (2016) 034 [arXiv: 1510.06930] [INSPIRE].

[69] I. Bandos, L. Martucci, D. Sorokin and M. Tonin, Brane induced supersymmetry breaking and de Sitter supergravity, JHEP 02 (2016) 080 [arXiv:1511.03024] [INSPIRE].

[70] I. Bandos, M. Heller, S.M. Kuzenko, L. Martucci and D. Sorokin, The Goldstino brane, the constrained superfields and matter in $\mathcal{N}=1$ supergravity, JHEP 11 (2016) 109 [arXiv: 1608.05908] [INSPIRE]. 